\title{
Probiotics in young and newborn animals
}

\author{
Denise Kelly \\ Rowett Research Institute \\ Greenburn Road, Bucksburn AB21 9SB, Aberdeen, Scotland
}

\begin{abstract}
The generation of "natural microbial products" which promote the barrier effect of the intestine and which are efficacious in the treatment of enteric infections, remains a critical target. To date, lactic acid bacteria have been the focus of much research and product development. Recent technical developments in molecular microbiology have identified other probiotic strains which may have greater potential for enhancing disease resistance. New research on the mechanisms of bacterial/ mucosal interactions will define their mode of action in the neonatal gut and provide a validation of the probiosis concept.
\end{abstract}

KEY WORDS: probiotics, commensal bacteria, intestine, oligosaccharides, receptors, immunology

\section{INTRODUCTION}

The intestinal microflora has, for decades, been considered to confer significant health benefits to the host and to play an important role in mucosal defence. The scientific impetus for the research activity on the "probiotic potential" of the indigenous microflora was based largely on work that established that germ-free and antibiotic-treated animals are significantly more susceptible to enteric infection compared to their conventionally-reared counterparts. The commercial impetus, which significantly augmented research funding aimed at identifying and marketing "probiotic microbials", was a direct result of the growing concern over the use of antibiotics as feed additives, their inevitable ban and the need for an efficacious, healthier, risk-free alternative. Marketing forces have in fact fueled the development and marketing of probiotics for many applications including feed additives for young and growing animals. The probiotic market has grown largely in the absence of critical scientific or clinical evaluation. The current scientific documentation describing the probiotic attributes of the commensal microflora, at best, perpetuates the claims that commensal bacteria confer functional benefits to 
the host. However, it does little to establish the principles or mechanisms governing bacteria/host cell interactions and completely fails to validate probiotics. There is now a need for high quality research which presents conclusive scientific evidence of probiotic effects on human and animal health. This will require a departure from near-market, product-orientated research and a refocusing on the fundamental principles of microbial ecosystems and host/bacterial interactions and crosstalk. This approach will provide a validation of probiosis and define bacterial strains with therapeutic functions. Given the recognised plight of antibiotics, this rescarch challenge is now a requisite.

\section{COLONISATION AND MANIPULATION OF THE INTESTINAL MICROFLORA}

The intestinal tract is sterile until birth. After birth the intestine is rapidly colonised by bacteria of both maternal and environmental origin. Despite the diversity in bacterial populations in the environment, the intestinal microflora of the newborn of most species is relatively simple and composed primarily of coliforms and streptococci (Conway, 1996). Obligate anacrobes, including Bacteroides, Eubacterium and Clostridium, colonise the gut a few days later. The sequence of microbial succession, in genus terms, appears to be predetermined and is remarkably similar across species (revicw by Conway, 1996). The microflora, which is estimated to contain several hundred different bacterial species, eventually attains a "stable" composition and it is this stability that has been suggested to confer colonisation resistance towards pathogens and to enhance host protection. The intestinal microflora, although stable, is dynamic and can be perturbed by alterations in the intestinal milieu. Such environmental changes can be generated by stress, antibiotic administration or by a dietary shift, such as occurs at weaning. The microbial imbalance associated with weaning has been correlated with an increased risk of enteric infection. Restoration of the microbial balance of the intestine is the basic tenet of probiotic therapy. The susceptibility of young animals to infection, both before and after weaning, is also attributable to poorly developed non-immune and immune barrier functions. Probiotics have therefore been used as prophylactics to stabilise the intestinal microflora and to promote natural defence mechanisms. Manipulation of the intestinal flora by administration of probiotics, i.e. "a live microbial feed supplement which beneficially affects the host animal by improving its intestinal microbial balance" (Fuller, 1992) has proved difficult and predictably, in vivo efficacy testing of probiotics have generated conflicting results. Variations in the bacterial strains selected, in their viability and in the health status of the host are likely to contribute towards the inconsistencies. However, the difficulty associated with introducing a new bacterial strain into an established or "stable" ecosystem is now fully recognised and perhaps represents one of the most 
significant problems in the development of efficacious probiotic products. There are several established microhabitats within the intestine including villus surface, crypts and luminal mucus which exert a selective influence on the composition and metabolic activity of the microflora. Within a niche, the composition can be further modulated by local environmental factors such as inter-bacterial competitions, nutrient composition, digesta flow, $\mathrm{pH}$, available molecular oxygen and the presence of gut receptors (Kelly et al., 1994). Logic would therefore suggest that to successfully introduce a probiotic strain into an established ecosystem, a microniche must be created. This requires that the triad of interactions between the gut, specific bacteria and the diet are appropriately defined. As an illustrative example, oral administration of Bifidobacterium breve to rats has been shown to result in a marked recovery of the fed strain from the caecum only when TOS (a mixture of $\beta-1,3,1,4$ and 1,6 linked glucose and galactose) is included in the diet (Rowland and Tanaka, 1993). This work serves to highlight the dynamic nature of the intestinal flora and the potential to modulate bacterial composition by dietary substrate.

Accordingly, dietary manipulation has proved to be a successful mcans whereby the composition of the intestinal microflora of the neonate and adult gut can be controlled or manipulated (Gibson and Roberfroid, 1995). Non-digestible food ingredicnts such as dictary fibre or oligosaccharides, which are hydrolysed to varying degrees and digested by colonic bacteria, are claimed to selectively stimulate the growth and /or activity of a number of bacterial strains (Gibson and Roberfroid, 1995; Djouzi and Andricux, 1997). Oligosaccharides, including raffinose, stachyose, fructo-oligosaccharides, isomalto-oligosaccharides and galacto-oligosaccharides are now being commercially exploited as "prebiotics" to promote a "healthy" intestinal flora. The addition of TOS to the diet of germ-free rats, inoculated with a suspension of human faecal flora, has been shown to significantly increase the numbers of viable anaerobic bacteria, bifidobacteria and lactobacilli in the faeces and reduce the numbers of E. coli (Rowland and Tanaka, 1993). Similarly, fructo-oligosaccharides have been reported to selectively modify the composition of the colonic microflora by stimulating the proliferation of bifidobacteria (Roberfroid, 1997). Although, there is an increasing opinion supporting the health benefits of oligosaccharides and the preferential growth of bifidobacteria and other lactic-acid producing strains, there is currently no scientific or clinical evidence to substantiate this. Unfortunately, as with probiotics, the marketing of prebiotics has preceded product validation.

\section{PROBIOTIC BACTERIA}

Of the vast number of commensal bacteria making up the complex microbial ecosystem of the gut, the probiotic actions of lactic acid-producing bacteria (LAB) 
have received unrivalled attention. The interest in $\mathrm{LAB}$ probably stems from properties such as their predominance within the microflora, the historical perception of health-links and importantly the observation that they are rarely pathogenic and thus have GRAS (gencrally recognised as safe) status. Efforts to devise effective probiotic preparations have relied on species or strains of LAB which are known to be both acid resistant and active colonisers of the gut (Kleeman and Klaenhammer, 1982; Mayra-Makinen et al., 1983). For example, three new Lactobacillus strains have recently been isolated, which exhibit acid and bile resistance, adhesion to various mucosal epithelia, coaggregation activity and also significantly suppress the growth of E. coli and Salmonella enteritidis in broth co-cultures (Drago et al., 1997). In addition to the aforementioned "desirable" probiotic characteristics, the ability of probiotic strains to display resistance to anti-microbial feed additives is also considered an important feature (Nemcova et al., 1997). There are examples where LAB have been tested in vivo but the results have been variable. For example, Streptococcus faecium was found to protect young rabbits from $E$. coli diarrhoea when given as a prophylactic agent at high dose (Wadstrom, 1984). In contrast, Lactobacillus spp. and Streptococcus faecium were found to be ineffective in preventing mortality and clinical symptoms in a weaned pig $E$. coli infection model (De Cupere et al., 1992). The in vivo effect of these organisms has not been demonstrated convincingly and the factors determining biological efficacy remain poorly defined.

Bacterial populations within the intestine exhibit great diversity, suggesting that strains other than the traditional LAB may have bencficial probiotic properties. Such strains are likely to exhibit properties which facilitate their colonisation within distinct regions or microhabitats within the gut. Molecular tracking, using 16Sr DNA technology, has already proven to be invaluable in defining the regional composition of the intestinal flora (jejunal/colonic and adherent/luminal). The results from the molecular-based strategy have been found to be at variance with data obtained using conventional microbiology (H. Flint, unpublished) and significantly, strains which have hitherto not been identified or cultured have been detected. Such bacterial strains may represent more efficacious or bioactive members of the microflora and their probiotic action may be very important. Bacteroides and Eubacterium arc numerically very significant and hence represent alternative candidates with potentially greater probiotic efficacy.

\section{MODE OF ACTION OF PROBIOTICS}

The beneficial claims for probiotics include growth-promotion, anti-pathogenic, anti-carcinogenic, anti-allergenic and anti-mutagenic effects (Renner and Munzner, 1991; Drago et al., 1997; Majamaa and Isolauri, 1997; Singh et al., 1997). The 
anti-pathogen mechanisms are particularly relevant to young animals. These mechanisms can be broadly categorised as direct bacterial antagonisms, competitive exclusion and immune modulation.

\section{BACTERIAL ANTAGONISMS}

The commensal flora produce a number of inhibitory substances which curtail the growth of other bacteria including enteric pathogens and therefore represent an important defence against microorganisms. These substances include organic acids, hydrogen peroxide and non-peptide or polypeptide antibiotics (bacteriocins). Bacteriocins have been investigated for decades and represent a very heterogeneous group of substances with different structural and biochemical properties. Despite the diversity of these substances their mode of action appears to be more uniform and involves permeabilisation of the cell membrane of target cells. A number of broad and narrow spectrum bacteriocins have recently been isolated and include Lacticin isolated from Lactococcus lactis (McAuliffe et al., 1998), Colicin U from Shigella Boydii (Smajs et al., 1997), Thermophilin 13 from Streptococcus thermophilus (Marciset et al., 1997) and Acidocin from Lactobacillus acidophilus (Tahara and Kanatani, 1996). The commercial exploitation of the antimicrobial activity of bacteriocins is obviously of interest. However, in view of the fact that the mode of action of bacteriocins is similar to that of therapeutic antibiotics, the issue of antibiotic resistance genes may well apply to bacteriocins.

\section{COMPETITIVE EXCLUSION}

Competitive exclusion is perhaps the most favoured hypothetical mode of action of probiotics but has proved particularly difficult to demonstrate in vivo. The rationale of competitive exclusion is that the attachment of pathogenic bacteria to the gut, which is achieved following ligation of fimbrial adhesins to specific gut receptors, can be prevented by adherent commensal bacteria with complementary receptor specificity. For example, there are reports of Lactobacillus strains which produce proteinaceous components that prevent attachment of $E$. coli to pig ileal mucus in vitro (Blomberg et al., 1993). This effect has not been demonstrated in vivo. There is generally a poor appreciation of the fact that pathogens have evolved very diverse mechanisms of attachment, involving multiple flagellae and adhesins with differing receptor specificities (Burns-Keliher et al., 1998). Furthermore, following the appropriate environmental stimulus, pathogenic bacteria display the capacity to induce the expression of distinct surface adhesive proteins (Bermudez et al., 1997; Burns-Keliher et al., 1998). Specifically, the composition of gut lumi- 
nal contents has been suggested to play an important role in determining the invasiveness and hence outcome of naturally acquired Salmonella infections (Clark et al., 1998). To further complicate the story, conventional animals and humans also exhibit a natural genotypic diversity, particularly in carbohydrate structures expressed on the surface of the gut epithelium. These carbohydrate epitopes are key determinants of pathogen recognition. Hence, the idea of presenting a "standard microbial probiotic preparation" to an animal or human population to target and eliminate an enteric pathogen(s) can only be viewed as naive.

In theory, targeting pathogens by competitive exclusion using chemical probiotics is a more feasible approach. For example, soluble non-toxic lectins may be administered where their carbohydrate specificities are selected to compete for the glycosyl binding sites of the fimbrial adhesin. This approach is exemplified by the use of GNA, the snowdrop lectin, which is highly specific for 1,3a linked D-mannose units, to block the proliferation of type-1 fimbriated E. coli (Pusztai et al., 1993). Equally, the in vitro attachment of bacteria to cell surfaces can be effectively inhibited by oligosaccharides of appropriate structure (Sharon et al., 1981; Oyofo et al., 1988). The efficacy of milk glycoproteins in protecting young pigs against infection by pathogenic $E$. coli has recently been demonstrated (Jeyasingham et al., 1996). However, the versatility and adaptibility of pathogens may well reduce the effectiveness of these approaches.

\section{IMMUNE MODULATION}

In addition to the direct interactions with pathogenic bacteria, certain members of the commensal flora are thought to improve disease resistance of the host by modulating systemic and mucosal immunity. In the healthy intestine, immune tolerance exists to the intestinal flora mainly as a result of appropriate Th2-mediated immune responses (Sudo et al., 1997). However, members of the flora are now recognised to modulate immune responses to foreign antigens, including bacteria. The best studied group in this regard are again the Lactobacilli. Lactobacillus strains have been shown to enhance the sIgA and serum IgG responses and to function as adjuvants in vaccination strategies (Perdigon et al., 1995; Pouwels et al., 1996). Furthermore, mice fed with $L$. casei were found to be resistant to a $S$. typhimurium challenge and this resistance was found to correlate closely with elevated levels of IgA in intestinal fluid (Perdigon et al., 1995). The effects of Lactobacillus strains, and undoubtedly other bacteria, are complex. It is now clear that administration of Lactobacillus strains can produce differential effects on the immune system which are strain-specific and that these effects are very sensitive to parameters, that in the past may have seemed trivial, such as the log phase of growth of the bacterium and the nature of the culture media (Maassen et al., 1997). 
Noteworthy are the segmented filamentous bacteria (SFB). This group of bacteria have been reported to colonise the small intestine of virtually all livestock (Smith, 1997). SFB are in intimate contact with their hosts and attach to the intestinal epithelium using a special holdfast as an anchor and are now recognised as commensals. SFB have been shown to increase intestinal levels of $\operatorname{IgA}$ and to promote proliferation of intraepithelial lymphocytes (Klassen et al., 1993; Snel et al., 1997). The induction of MHC class II molecules and fucosyl asialoglycolipids on epithelial cells has also been documented (Umesaki et al., 1995). Although reports on the biological activities of these bacteria are few in number, the above studies suggest that these bacteria may play an important role in regulating intestinal function and immune resistance and hence are worthy of further investigation.

\section{CONCLUSIONS}

Health benefits, particularly enhanced discase resistance, have been associated with probiotics for almost a century. However, scientific and clinical validation is inadequate. The future development of probiotics is dependent on robust research which, from the microbiological point of view, results in the identification of efficacious commensal probiotics. The probiotic function of organisms other than LAB is likely to be significant. Development of bacterial strains which carry multiple probiotic traits using gene technology is likely to be an important future goal. Investigation of natural bacterial components which govern the selection of these bacteria within the context of the gut milieu and the presence of other competing organisms is also important. From the host view point, understanding how bacteria influence host immune responses in health and discase is perhaps the most significant challenge. The nature of the bacterial constituents which trigger such host responses may result in the generation of important immunomodulators.

\section{REFERENCES}

Bermudez L.E., Petrofsky M., Goodman J., 1997. Exposure to low oxygen tension and increased osmolarity enhance the ability of Mycohacteritum avium to enter intestinal epithelial (HT29) cells. Infec. Immunity 65, 3768-3773

Blomberg L., Henriksson A., Conway PL., 1993. Inhibition of adhesion of Escherichia coli K88 to piglet ileal mucus by Lactobacillus spp. Appl. Environ. Microbiol. 59, 34-39

Burns-Keliher L., Nickerson C.A., Morrow B.J., Curtiss R., 1998. Cell-specific proteins synthesised by Salmonella typhimurium. Infec. Immunity 66, 856-861

Clarke M.A., Hirst B.H., Jepson M.A., 1998. Inoculum composition and salmonella pathogenicity island 1 regulate $\mathrm{M}$ cell invasion and epithelial destruction by Salmonella typhimurium. Infec. Immunity $66,724-731$ 
Conway P.L., 1996 Development of Intestinal Microbiota. In: R.I. Mackie, B.A. Whyte, R.E. Isaacson (Editors). Gastrointestinal microbiology Vol 2. Gastrointestinal microbes and host interactions. Chapman and Hall, pp. 3-38

De Cupere F., Deprez P., Demeulenaere D., Muylle E., 1992. Evaluation of the effect of three probiotics on experimental E. coli enterotoxiaemia in weaned pigs. Zbl. Vetmed, 39, 277-284

Drago L., Gismondo M.R., Lombardi A., De Haen C., Gozzini L., 1997. Inhibition of in vitro growth of enteropathogens by Lactobacillus isolates of human intestinal origin. FEMS Microbiol. Lett. $153,455-463$

Djouzi Z., Andrieux C., 1997. Compared effects of three oligosaccharides on the metabolism of intestinal microflora in rats inoculated with a human faecal flora. Brit. J. Nutr. 78, 313-324

Fuller R., 1992 History and development of probiotics. In: R. Fuller (Editor). Probiotics the scientific basis. London Chapman and Hall. pp. 1-8

Gibson G.R., Roberfroid M.B., 1995. Dietary modulation of the human colonic microbiota: introducing the concept of prebiotics. J. Nutr. 125, 1401-1412

Jeyasingham M.D., Begbie R., King T.P., Kelly D., 1996. Inhibition of K88 E. coli by bovine milk glycoproteins. Rowett Ann. Rep., pp. 29

Kelly D., Begbie R., King T.P., 1994. Nutritional influences on interactions between bacteria and the small intestinal mucosa. Nutr. Res. Rev. 7, 233-257

Klassen H.L.B.M., Van der Heijden W., Stok J.P., Koopman M.E., 1993 Apathogenic, intestinal, segmented, filamentous bacteria stimulate the mucosal immune system of mice. Infec. Immunity $61,303-306$

Kleeman E.G., Klaenhammer T.R., 1982. Adherence of Lactobacillus species to human fetal intestinal cells. J. Dairy Sci. 65, 2063-2069

Majamaa H., Isolauri E., 1997. Probiotics: a novel approach in the management of food allergy. J. Allerg. Clin. Immunol. 99, 179-185

Marciset O., Jeronimus-Stratingh M.C., Mollet B., Poolman B., 1997. Thermophilin 13, a nontypical antilisterial poration complex bacteriocin, that functions without a receptor. J. Biol. Chem. $272,14277-14284$

Maassen C.B.M,, Van Holten J.C.A.M., Balk F., 1997. Orally administered Lactobacillus strains differentially affect the direction and efficacy of the immune response. Proceedings of the World Congress on Food Hygiene. The Hague, The Netherlands, pp. 62-66

Mayra-Makinen A., Manninen M., Gyllenberg H., 1983. The adherence of lactic acid bacteria to the columnar epithelial cells of pigs and calves. J. Appl. Bacteriol. 55, 241-245

McAuliffe O., Ryan M.P., Ross R.P., Hill C., Breeuwer P., Abee T., 1998. Lacticin 3147, a broadspectrum bacteriocin which selectively dissipates the membrane potential. Appl. Environ. Microbiol. 64, 439-445

Nemcova R., Laukova A., Gancarcikova S., Kastel R., 1997. In vitro studies of porcine lactobacilli for possible probiotic use. Berl. Mun. Tierarztl. Wochenschr. 110, 413-417

Perigon G., Alvarez S., Rachid M., Aguero G., Gobbato N., 1995. Immune system stimulation by probiotics. J. Dairy Sci. 78, 1597-1606

Pouwels P.H., Leer R.J., Bocrsma W.J.A., 1996. The potential for lactobacillus as a carrier for oral immunisation. Development and preliminary characterisation of vector systems for targeted delivery of antigens. J. Biotech. 44, 183-192

Pusztai A., Grant G., Spencer R.J., 1993. Kidney-bean lectin-induced E. coli overgrowth in the small intestine is blocked by GNA, a mannose-specific lectin. J. Appl. Bacteriol. 75, 360-368

Renner H.W., Munzner R., 1991. The possible role of probiotics as dictary antimutagen. Mutat. Res. $262,239-245$ 
Roberfroid M.B., 1997. Health benefits of non-digestible oligosaccharides. Advan. Exp. Med. Biol. 427, $211-219$

Rowland I.R., Tanaka R., 1993 The effects of transgalactosylated oligosaccharides on gut flora metabolism in rats with a human faecal flora. J. Appl. Bacteriol. 74, 667-674

Sharon N., Eshdat Y., Silverblatt F.J., Ofek I., 1981. Bacterial adherence to ceil surface sugars. In adhesion and Micro-organisms Pathogenicity. K. Elliot, M. O'Connor, J. Whelan (Editors). Pitman Press, London, pp. 119-141

Singh J., Riverson A., Tomita M., Shimamura S., Ishibashi N., Reddy B.S., 1997. Bifidobacterium longum, a lactic acid-producing intestinal bactcrium inhibits colon cancer and modulates the intermediate biomarkers of colon carcinogenesis. Carcinogenesis 18, 833-84I

Smajs D., Pilsl H., Braun V., 1997. Colicin U, a novel colicin produced by Shigella boydii. J. Bacteriol. $179,4919-4928$

Smith T.M., 1997. Segmented filamentous bacteria in the bovine small intestine. J. Comp. Pathol. $117,185-190$

Snel J., Bakker M.II., Heidt P.J., 1997. Quantification of antigen-specific immunoglobulin A after oral booster immunisation with ovalbumin in mice mono-associated with segmented Filamentous bacteria or Clostridium innocuum. Immunol. Lett. 58, 25-28

Sudo N., Sawamura S., Tanaka K., Aiba Y., Kubo C., Koga Y., 1997. The requirement of intestinal bacterial flora for the development of an IgE production system fully susceptible to oral tolerance induction. J. Immunol. 159, 1739-1745

Tahara T., Kanatani K., 1996. Isolation, a partial characterisation and mode of action of acidocin J1229, a bacteriocin produced by Lactobacillus acidophilus JCM1229. J. Appl. Bacteriol. 81, 669-677

Umesakai Y., Okada Y., Matusumoto S., Imaoka A., Setoyama H., 1995. Segmented filamentous bacteria are indigenous intestinal bacteria that activate intraepithelial lymphocytes and induce MHC II molecules and fucosyl asialo GM1 glycolipids on the small intestinal epithelial cells in the ex-germ free mouse. Microbiol. Immunol. 39, 555-562

Wadstrom T., 1984. Streptococcus faecium M74 in control of diarrhoea induced by a human enterotoxigenic E. coli strain in an infant rabbit model. Zbl. Bakteriol. Mikrobiol. Hyg. 257, 357-363 